\title{
Classification of Congenital Heart Disease by SVM-MFCC Using Phonocardiograph
}

\author{
Gholamreza Attarodi ${ }^{1}$, Asghar Tareh ${ }^{1}$, Nader Jafarnia Dabanloo ${ }^{1}$, \\ Ali Adeliansedehi ${ }^{2}$ \\ ${ }^{1}$ Islamic Azad University, Science and Research Branch, Tehran, Iran \\ ${ }^{2}$ Tehran Medical University, Tehran, Iran
}

\begin{abstract}
In this paper, a new method is presented for nonlinear processing and classification of congenital heart valveseptum diseases in neonates. Two main groups of congenital heart diseases in neonates are aortic valve stenosis, and inter-ventricular septum puncture. Both diseases create harsh sounds in the heart's first sound area, S1. In this study, using a compilation of MFCC (Mel-frequency cepstral coefficient) and Auto Correlation methods, we separated the $1^{\text {st }}$ sound range with high precision and thereafter managed to classify three groups of neonates: with normal sound, murmur sound resulted by VSD (ventricular septum defect), and murmur sound resulted by AS(aortic stenosis) using SVM(support vector machine) classifier equipped with RBF (radial-basis function) and Quadratic kernels. With regard to the data distribution in the feature space that was based on short term energy of 32-fold time intervals of wavelet transform's level 2 coefficients with db kernel, the SVMQuadratic classifier managed to classify the three groups of foresaid neonates with $78 \%$ precision and SVM-RBF classifier with $96 \%$ precision.
\end{abstract}

\section{Introduction}

In order to eliminate the noise of signals in various papers, various methods have been used based on discrete wavelet coefficients. In the sequel, after clearing the signal by wavelet transform, to extract the first and second sounds and make a distinction between them, we sought the best way to separate the first and second sounds. According to our previous articles, the best way to extract the first and second heart rate range is to use the
MFCC method along with the auto correlation (MFCC_AC) method. In the MFCC method, after the use of a cepstral transform, the envelope of the phonocardiograph was extracted and using autocorrelation method the first sound of heart was extracted. The reason for our obligation to extract the first sound is the effect of both illness in the first sound. After that, for extracting the signal characteristics using the wavelet transform, a feature vector was created for each patient, and ultimately, due to the complexity and non-linear properties of the space, the SVM classifier was selected.Fig.1 illustrates the stages of the above-said Process.

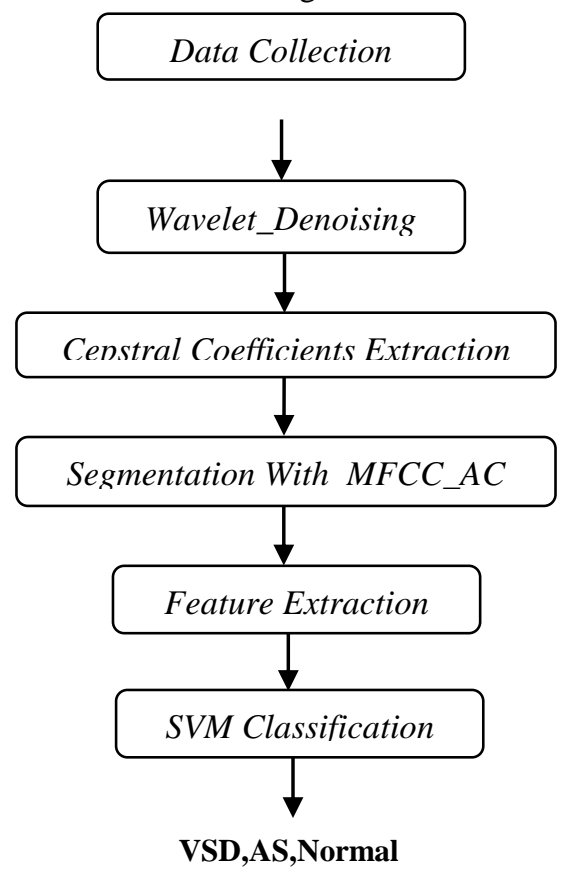

Fig. 1 


$$
\left.R\right|_{x x}(m)= \begin{cases}\sum_{n=0}^{N-m-1} x(n+m) x(n) & m \geq 0 \\ R(-m) & m<0\end{cases}
$$

\section{Wavelet denoising}

Since the existence of some soufflés and murmurs in PCG signal prevented us to consider this signal as a nondeterministic or non-stationary signal. Therefore, the methods such as signal averaging will make no favorable results[1]; thus we used the noise removal method based on the wavelet (in this method we used the command 'wden' in MATLAB software).The correct selection of cases such as type of Wavelet, number of decomposition levels, and thresholding method is necessary. Using a wavelet with 5 levels will make favorite results. Also, we used the 'Regrsure' thresholding method in order to select threshold for removing noise.

\section{Segmentation with MFCC_AC}

After removing the noise, the cepstral coefficient of each signal was extracted through the process of Fig. 2 below:

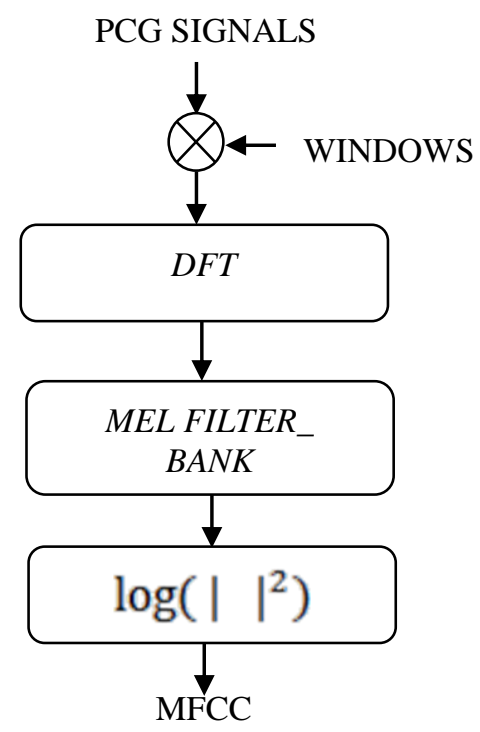

Fig. 2

To complete the auto correlation separation step, the second-order cepstral coefficients of the signal were calculated according to the following equation:
Fig.3 shows a sample of the signal and its filtered with its envelope signal and its auto correlation:

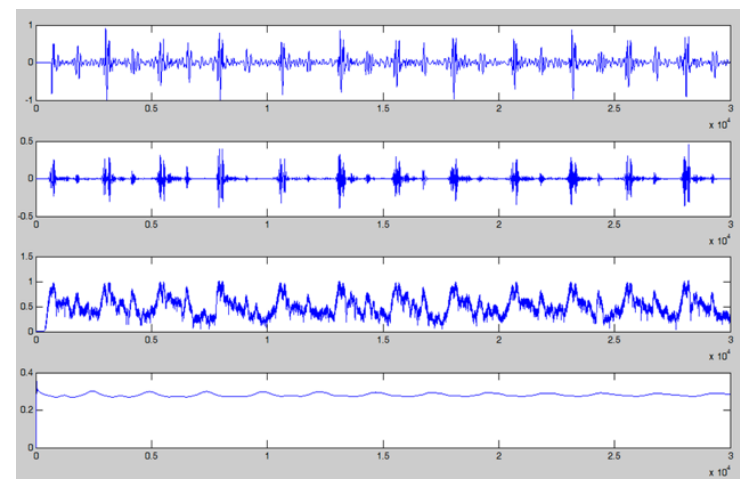

Fig. 3

Eventually, by finding the maximum amount of auto correlation in the range of 1000 to 5000 , the length of a period of the signals was extracted from each 5-period signal. Fig.4 shows the following:

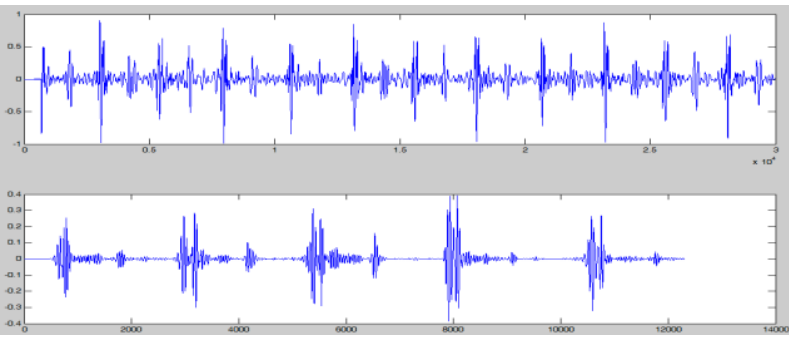

Fig. 4

\section{4.}

\section{Feature Extraction}

In the following, a discrete wavelet was used to extract the features. By reviewing multiple articles, we used $\mathrm{db}_{-}$ wavelet and duplicate Decomposition levels. Then the coefficients for the second detail were selected. To construct the feature vector, we divide the entire interval 
d2 into 32 non-overlapping sections and calculate its short-term energy according to Parseval. Subsequently, using the Min-Max method, the extraction features were normalized. Thus, for each patient, the feature vector was constructed with 32 dimensions.

\section{Result}

The property of the acquired feature space as well as the number of features leads to the use of powerful classifiers such as SVM. It is obvious that the liner SVM kernels are not able to differentiate between classes with complex distribution in the space of the property. So the results of the work show that the classification accuracy with linear kernels is far lower and will not have much value. But in nonlinear kernels such as RBF and quadratic function, satisfactory results are presented. In this study, the nonlinear kernel of the quadratic function was $78 \%$ accurate and the nonlinear RBF kernel with a precision of $96 \%$ of the three groups of patients was classified.

\section{References}

[1] Syed Z, Leed D, Curthis D, Nesta F, Levin RA, Guttag J: A Framework for the Analysis of Acoustical Cardiac Signals.IEEE Transactions on Biomedical Engineering 2007, 1(51):651-662..

[2] Liang H, Lukkarinen S, Hartimo I: Heart Sound Segmentation Algorithm Based on Heart Sound Envelograms.Computers in Biology 1997, 24: 105-108.P.

[3] Gupta CN, Palaniappan R, Swaminathan S, Krishnan SM: Neural Network Classification of Homomorphic Segmented Heart Sound. Applied Soft Computing 2007, 7: 286-297.

[4] Kumar D, Carvalho P, Antunes M, Henriques J, e Melo AS, Habetha J: Heart Murmur Recognition and Segmentation by Complexity Signatures. In Proceedings of the 30th IEEE EMBS Annual Conference 2008.

\section{Contact Information}

Gholamreza Attarodi

Postal Address: Flat 1,No.4, Booyeh St.Shariati, Gholhak 1916773511 Tehran, Iran

Email Address: attarodi@gmail.com 
Page 4 